\title{
A Linear Singularity Analysis by the Continuous Shearlet Transform
}

\author{
Songkiat Sumetkijakan \\ Department of Mathematics, Faculty of Science, Chulalongkorn University \\ Phyathai Road, Patumwan, Bangkok 10330, Thailand \\ and \\ Centre of Excellence in Mathematics, CHE \\ Si Ayutthaya Rd., Bangkok 10400, Thailand \\ songkiat.s@chula.ac.th
}

\begin{abstract}
Several authors have related the linear singularities of two-variable functions to the decays of their parabolic transforms. Kutyniok and Labate computed the decay rates of the continuous shearlet transform of certain point, linear and corner singularities. Modelling the linear singularity of a function by two (non-parallel) directional Hölder exponents, Lakhonchai, Sampo and Sumetkijakan obtained decay estimates of its shearlet transform. They found that the decay estimates for shearlets whose major axes are close to the singularity line are proportional to the Hölder exponent in the direction of the singularity line. Likewise, the decay estimates for the other shearlets are proportional to the the Hölder exponent in a nonparallel direction. In this paper, we improve Lakhonchai et al.'s result by narrowing the gap between the decay estimate and the Hölder exponent in the case of the non-parallel direction. As a result, we obtained the decay rates of a linear singularity model reminiscent to that of Kutyniok et al.
\end{abstract}

Key words and phrases: Directional Hölder regularity, Shearlet transforms, Parabolic scaling, Direction of singularity, Linear singularity

2000 AMS Mathematics Subject Classification - 26B35, 42B10

\section{Introduction}

Almost as soon as wavelet transform became popular, various transforms have been studied with respect to their abilities to detect edges. This direction of research is mainly due to the characterizations of point singularity by the continuous wavelet transform, discovered independently by Jaffard [19] and Holschneider and Tchamitchian [18] in the early 1990's. These transforms included the Smith transform [33], curvelet transform [2,3] and, the most recently introduced, the shearlet transform [25]. 
Due partly to their success in edge detection, an attempt to use the Smith and continuous curvelet transforms to characterize pointwise and uniform Hölder regularities has been reported [32]. The more interesting result of their study concerned how a set of directional and local Hölder regularities yielded the decay estimates of the transforms. Though the converse is not at all plausible, they managed to prove that these decay estimates imply pointwise regularity of certain exponents.

Based on a beautiful theory of wavelets with composite dilations [16, 17], the shearlet system is defined [10] in the time domain by translations, parabolic dilations, and shearing of a single admissible function, and have a simple reconstruction formula. Kutyniok and Labate [25] computed the decay rates of continuous shearlet transforms when the distributions possess point, linear, polygonal or circular singularities. Independently, Lakhonchai, Sampo and Sumetkijakan [27] gave estimates of the continuous shearlet transforms of functions satisfying a set of directional Hölder regularities and so which models a linear singularity.

In this paper, we first give an improved version of the theorem of Lakhonchai et al. (Theorem 5.3 in [27]) with a weaker condition. The same argument works just as well in relaxing the $C^{2 \alpha+1+\varepsilon}$-condition of Sampo and Sumetkijakan (Theorem 8 of [32]) where continuous curvelet and Hart Smith transforms are used. In light of the analysis of linear singularities of a linear delta distribution in [25], we present an analysis of the linear singularities of our model. As a corollary, in the direction of the singularity line, the decay rate of the continuous shearlet transform corresponds precisely to the Hölder exponent in the horizontal direction at the same point, while the decay rate of the transform in other directions corresponds to the Hölder exponent in the direction of the singularity line.

\section{Basics of Hölder Regularity and Shearlets}

Points in the time domain are denoted by column vectors $\boldsymbol{x}=\left(x_{1}, \ldots, x_{d}\right)^{\mathrm{T}}$ while points in the frequency domain are denoted by row vectors $\boldsymbol{\xi}=\left(\xi_{1}, \ldots, \xi_{d}\right)$, such that $\boldsymbol{\xi} \boldsymbol{x}=\sum_{i=1}^{d} \xi_{i} x_{i}$. The Fourier transform of $f \in L^{1}\left(\mathbb{R}^{d}\right)$ is defined by $\hat{f}(\boldsymbol{\xi})=\int_{\mathbb{R}^{d}} f(\boldsymbol{x}) e^{-2 \pi i \boldsymbol{\xi} \boldsymbol{x}} d \boldsymbol{x}, \boldsymbol{\xi} \in \mathbb{R}^{d}$ and the Fourier transform of an $L^{2}$-function is defined in the limit. So the Plancherel's formula becomes $\langle f, g\rangle=\langle\hat{f}, \hat{g}\rangle$ and the Fourier inversion formula is $f(\boldsymbol{x})=\int_{\widehat{\mathbb{R} d}} \hat{f}(\boldsymbol{\xi}) e^{2 \pi i \boldsymbol{\xi} \boldsymbol{x}} d \boldsymbol{\xi}$.

Pointwise, uniform, and directional Hölder regularities of functions of several variables are defined as follows.

Definition 1. Let $\alpha \in(0, \infty) \backslash \mathbb{N}$ and $\boldsymbol{u} \in \mathbb{R}^{d}$. The function $f: \mathbb{R}^{d} \rightarrow \mathbb{R}$ is said to be pointwise Hölder regular with exponent $\alpha$ at $\boldsymbol{u}$, denoted by $f \in C^{\alpha}(\boldsymbol{u})$, if there exists a polynomial $P=P_{\boldsymbol{u}}$ of a degree less than $\alpha$ and a constant $C=C_{\boldsymbol{u}}$ such that for all $\boldsymbol{x}$ in a neighborhood of $\boldsymbol{u}$

$$
|f(\boldsymbol{x})-P(\boldsymbol{x}-\boldsymbol{u})| \leqslant C\|\boldsymbol{x}-\boldsymbol{u}\|^{\alpha} .
$$


Let $\Omega$ be an open subset of $\mathbb{R}^{d}$. If there exists a uniform constant $C$ so that for all $\boldsymbol{u} \in \Omega$ there is a polynomial $P_{\boldsymbol{u}}$ of a degree less than $\alpha$ such that (1) holds for all $\boldsymbol{x} \in \Omega$, then we say that $f$ is uniformly Hölder regular with exponent $\alpha$ on $\Omega$ or $f \in C^{\alpha}(\Omega)$.

For a fixed unit vector $\boldsymbol{v} \in \mathbb{R}^{d}, f$ is said to be pointwise Hölder regular with exponent $\alpha$ at $\boldsymbol{u}$ in the direction $\boldsymbol{v}$, denoted by $f \in C^{\alpha}(\boldsymbol{u} ; \boldsymbol{v})$, if there exist a constant $C=C_{\boldsymbol{u}, \boldsymbol{v}}$ and a polynomial $P=P_{\boldsymbol{u}, \boldsymbol{v}}$ of a degree less than $\alpha$ such that (1) holds for all $\boldsymbol{x}$ in an open line segment that contains the point $\boldsymbol{u}$ and is parallel to $\boldsymbol{v}$.

Let $\Omega_{1}$ be a subset of $\mathbb{R}^{d}$ and $\Omega_{2}$ be an open neighborhood of $\Omega_{1}$. Then, $f$ is said to be in $C^{\alpha}\left(\Omega_{1}, \Omega_{2} ; \boldsymbol{v}\right)$ if there exists a constant $C=C_{\boldsymbol{v}}$ so that for all $\boldsymbol{u} \in \Omega_{1}$ there is a polynomial $P=P_{\boldsymbol{u}, \boldsymbol{v}}$ of a degree less than $\alpha$ such that (1) holds for all $\boldsymbol{x} \in \Omega_{2}$ on the line passing through $\boldsymbol{u}$ and parallel to $\boldsymbol{v}$. If $\Omega_{1}=\Omega_{2}$, then we denote $C^{\alpha}\left(\Omega_{1}, \Omega_{2} ; \boldsymbol{v}\right)$ by $C^{\alpha}\left(\Omega_{1} ; \boldsymbol{v}\right)$.

\subsection{Continuous Shearlet Transform}

Following Kutyniok and Labate [25], we choose the following definition of the continuous shearlet transform.

Definition 2. Given $\psi_{1}$ and $\psi_{2} \in L^{2}(\mathbb{R})$, let $\psi \in L^{2}\left(\mathbb{R}^{2}\right)$ be defined by

$$
\hat{\psi}(\boldsymbol{\xi})=\hat{\psi}_{1}\left(\xi_{1}\right) \hat{\psi}_{2}\left(\frac{\xi_{2}}{\xi_{1}}\right) \quad \text { for } \boldsymbol{\xi}=\left(\xi_{1}, \xi_{2}\right) \in(\mathbb{R} \backslash\{0\}) \times \mathbb{R} .
$$

Then $\psi$ is called a continuous shearlet function if:

i. $\psi_{1} \in L^{2}(\mathbb{R})$ satisfies the admissibility condition, that is

$$
\int_{-\infty}^{\infty} \frac{|\widehat{\psi}(\xi)|^{2}}{|\xi|} d \xi<\infty
$$

and $\hat{\psi}_{1} \in C^{\infty}(\mathbb{R})$ with supp $\hat{\psi}_{1} \subset\left[-2,-\frac{1}{2}\right] \cup\left[\frac{1}{2}, 2\right]$; and

ii. $\|\psi\|_{2}=1$, and $\hat{\psi}_{2} \in C^{\infty}(\mathbb{R})$ with $\operatorname{supp} \hat{\psi}_{2} \subset[-1,1]$ and $\hat{\psi}_{2}>0$ on $(-1,1)$.

A continuous shearlet system is the set of functions generated by $\psi$, namely,

$$
\left\{\psi_{\text {ast }}=a^{-\frac{3}{4}} \psi\left(\mathrm{M}_{a s}^{-1}(\cdot-\boldsymbol{t})\right): a \in(0,1), s \in[-2,2], \boldsymbol{t} \in \mathbb{R}^{2}\right\},
$$

where $\mathrm{M}_{a s}=\mathrm{B}_{s} \mathrm{D}_{a}, \mathrm{~B}_{s}$ is the shear matrix $\left(\begin{array}{cc}1 & -s \\ 0 & 1\end{array}\right)$ and $\mathrm{D}_{a}$ is the diagonal matrix $\left(\begin{array}{cc}a & 0 \\ 0 & \sqrt{a}\end{array}\right)$. The continuous shearlet transform of $f \in L^{2}\left(\mathbb{R}^{2}\right)$ is then defined by

$$
\mathcal{S} \mathcal{H}_{\psi} f(a, s, \boldsymbol{t})=\left\langle f, \psi_{\text {ast }}\right\rangle, \text { for } a \in(0,1), s \in[-2,2] \text { and } \boldsymbol{t} \in \mathbb{R}^{2} .
$$


Let $E \subset \widehat{\mathbb{R}^{2}}$ be given by $E=\left\{\left(\xi_{1}, \xi_{2}\right) \in \widehat{\mathbb{R}^{2}}:\left|\xi_{1}\right| \geqslant 2\right.$ and $\left.\left|\frac{\xi_{2}}{\xi_{1}}\right| \leqslant 1\right\}$ and define $L^{2}(\mathrm{E})=\left\{f \in L^{2}\left(\mathbb{R}^{2}\right): \operatorname{supp} \hat{f} \subset E\right\}$. Then, there is a reconstruction formula for functions in this proper subspace.

Theorem 2.1. Let $\psi \in L^{2}\left(\mathbb{R}^{2}\right)$ be a shearlet function. Then, for all $f \in L^{2}(E)$,

$$
f=\int_{\mathbb{R}^{2}} \int_{-2}^{2} \int_{0}^{1}\left\langle\psi_{\text {ast }}, f\right\rangle \psi_{\text {ast }} \frac{d a}{a^{3}} d s d \boldsymbol{t} \quad \text { in } L^{2}(E) .
$$

However, it is still possible to obtain a reproducing formula for all $f \in L^{2}\left(\mathbb{R}^{2}\right)$ by defining a vertical shearlet function $\psi^{(v)}$ by

$$
\widehat{\psi^{(v)}}(\boldsymbol{\xi})=\widehat{\psi^{(v)}}\left(\xi_{1}, \xi_{2}\right)=\hat{\psi}_{1}\left(\xi_{2}\right) \hat{\psi}_{2}\left(\frac{\xi_{1}}{\xi_{2}}\right)
$$

where $\hat{\psi}_{1}, \hat{\psi}_{2}$ are defined as in Definition 2 above. The shearlets $\psi_{\text {ast }}^{(v)}$ are defined by $\psi_{\text {ast }}^{(v)}=a^{-\frac{3}{4}} \psi\left(\left(\mathrm{M}_{a s}^{(v)}\right)^{-1}(\cdot-\boldsymbol{t})\right)$, where $\mathrm{M}_{a s}^{(v)}=\mathrm{B}_{s}^{(v)} \mathrm{D}_{a}^{(v)}$ with $\mathrm{B}_{s}^{(v)}=\mathrm{B}_{s}^{\mathrm{T}}$ and $\mathrm{D}_{a}^{(v)}=\left(\begin{array}{cc}\sqrt{a} & 0 \\ 0 & a\end{array}\right)$. Then $\left\{\psi_{a s t}^{(v)}\right\}$ is the continuous shearlet system for $L^{2}\left(E^{(v)}\right)^{\smile}$ where $E^{(v)}=\left\{\left(\xi_{1}, \xi_{2}\right) \in \widehat{\mathbb{R}^{2}}:\left|\xi_{2}\right| \geqslant 2\right.$ and $\left.\left|\frac{\xi_{2}}{\xi_{1}}\right|>1\right\}$, and the associated vertical continuous shearlet transform is

$$
\mathcal{S H}_{\psi}^{(v)} f(a, s, \boldsymbol{t})=\left\langle f, \psi_{\text {ast }}^{(v)}\right\rangle .
$$

A corresponding reconstruction formula holds for $f \in L^{2}\left(E^{(v)}\right)^{\smile}$. As a result, all functions whose frequency support is contained in $\widehat{R^{2}} \backslash(-2,2)^{2}$ can be reconstructed from the respective horizontal and vertical continuous shearlet transforms. Since bandlimited functions are necessarily $C^{\infty}$, these continuous shearlet transforms are sufficient for regularity analysis purposes. Moreover, as $\psi_{\text {ast }}$ and $\psi_{\text {ast }}^{(v)}$ have essentially the same decay properties and complimentary vanishing directional moments, we shall only focus on $\psi_{\text {ast }}$, but note that analogous statements for $\psi_{a s t}^{(v)}$ can be obtained in a similar fashion.

\subsection{Properties of Shearlet Functions}

Definition 3. The function $f$ of two variables is said to have an $L$-order vanishing directional moments along the direction $\boldsymbol{v}=\left(v_{1}, v_{2}\right)^{T} \neq \mathbf{0}$ if

$$
\int_{\mathbb{R}} b^{n} f(b \boldsymbol{v}+\boldsymbol{w}) d b=0, \quad \text { for all } \boldsymbol{w} \in \mathbb{R}^{2} \text { and } 0 \leqslant n<L .
$$

The following decay and vanishing directional moments of shearlet functions, taken from [27], are used heavily in our regularity analysis and so are shown below. 
Lemma 2.2. For all $a \in(0,1), s \in[-2,2]$ and $\boldsymbol{t} \in \mathbb{R}^{2}$, the following is true.

1. The shearlet functions $\psi_{\text {ast }}$ have vanishing directional moments of any order $L<\infty$ along any direction $\boldsymbol{v}=\left(v_{1}, v_{2}\right)^{\mathrm{T}}$ satisfying $\left|v_{2} s+v_{1}\right|>$ $\left|v_{2}\right| \sqrt{a}$.

2. For each $N=1,2, \ldots$, there is a constant $C_{N}$ independent of $a, s$ and $\boldsymbol{t}$ such that

$$
\left|\partial^{\nu} \psi_{a s t}(\boldsymbol{x})\right| \leqslant \frac{C_{N} a^{-3 / 4-|\nu|}(\sqrt{a}+|s|)^{\nu_{2}}}{1+\left\|\mathrm{D}_{1 / a} \mathrm{~B}_{-s}(\boldsymbol{x}-\boldsymbol{t})\right\|^{2 N}}
$$

for all $\boldsymbol{x} \in \mathbb{R}^{2}$ and $\nu \in \mathbb{N}_{0}^{2}$.

\section{The Main Results}

In this section, for any given $L>0, s_{0} \in[-2,2]$ and $\boldsymbol{u}=\left(u_{1}, u_{2}\right) \in \mathbb{R}^{2}$, let $\Gamma_{\boldsymbol{u}}$ denote the vertical line passing through $\boldsymbol{u}$ and let $\Gamma_{\boldsymbol{u}, s_{0}}$ denote the line passing through $\boldsymbol{u}$ with slope $-\frac{1}{s_{0}}$. Observe that we may write $\Gamma_{\boldsymbol{u}}=\Gamma_{\boldsymbol{u}, 0}$ so that $\left(x_{1}, x_{2}\right) \in \Gamma_{\boldsymbol{u}, s_{0}}$ if and only if $x_{1}=-s_{0}\left(x_{2}-u_{2}\right)+u_{1}$. Recall that for a given subset $N$ of $\mathbb{R}^{2}, N(L)$ denotes the $L$-neighborhood of $N$, i.e. the set of all points whose distance to $N$ is less than $L$.

For the purpose of comparison, we quote Theorem 5.3 from [27].

Theorem 3.1. Let $u_{1} \in \mathbb{R}, \alpha \in(0,1], L>0$, and $\varepsilon>0$. Suppose $f$ is a bounded function in $C^{\alpha}\left(\Gamma_{\left(u_{1}, 0\right)}, \Gamma_{\left(u_{1}, 0\right)}(L) ;(1,0)\right)$ and $C^{2 \alpha+1+\varepsilon}\left(\Gamma_{\left(u_{1}, 0\right)}(L) ;(0,1)\right)$. Then there exist $C<\infty$ and a fixed scale $a_{0}<1$ such that if $0<a<a_{0},-2 \leqslant s \leqslant 2$, and $\boldsymbol{t}=\left(t_{1}, t_{2}\right) \in \Gamma_{\left(u_{1}, 0\right)}(r)$ with $r<L / 2$, then

$$
\left|\left\langle\psi_{\text {ast }}, f\right\rangle\right| \leqslant \begin{cases}C a^{\alpha+\frac{3}{4}}\left(1+\left|\frac{t_{1}-u_{1}}{a}\right|^{\alpha}\right) & \text { if }|s| \leqslant \sqrt{a}, \\ C a^{\alpha+\frac{5}{4}} & \text { if }|s| \geqslant \sqrt{a} .\end{cases}
$$

In this theorem, the two main assumptions are (i) that the "local" directional Hölder regularity of $f$ on the line $x=u_{1}$ in the perpendicular direction is at least $\alpha$ and (ii) that its directional Hölder regularity on a neighborhood of the line $x=u_{1}$ in the parallel direction is at least $2 \alpha+1+\varepsilon$. The first $C^{\alpha}$ regularity assumption in the perpendicular direction implies a decay estimate of the order $\alpha+\frac{3}{4}$ for small values of the shear-scale ratio $(|s| \leqslant a)$, while the $C^{2 \alpha+1+\varepsilon}$ regularity assumption in the parallel direction implies a decay estimate of the order $\alpha+\frac{5}{4}$ for a large shear-scale ratio $(|s| \geqslant a)$. This means that the $C^{\beta+\varepsilon}$ regularity in the parallel direction would only imply a decay estimate of the order $(\beta-1) / 2$. Our investigation shows that the decay estimate resulting from the regularity assumption in the parallel direction can be improved for larger values of $|s|$. Even though there is no improvement in the other half of the Theorem, 
corresponding to the regularity in the perpendicular direction, we include its statement here in item 1 for completeness. Its proof, which can be found in [27], is naturally omitted.

Theorem 3.2. Let $0<\alpha \leqslant 1, \beta>0$ and $0<\varepsilon<1$ be such that $(1+\varepsilon) \beta \notin \mathbb{N}$. Suppose $f: \mathbb{R}^{2} \rightarrow \mathbb{R}$ is bounded and let $u_{1} \in \mathbb{R}$ and $L>0$ be given.

1. If $f \in C^{\alpha}\left(\Gamma_{\left(u_{1}, 0\right)}, \Gamma_{\left(u_{1}, 0\right)}(L) ;(1,0)\right)$ then there exists a constant $C<\infty$ such that for all $a \in(0,1), \boldsymbol{t} \in \mathbb{R}^{2}$ and $|s| \leqslant \sqrt{a}$,

$$
\left|\left\langle\psi_{\text {ast }}, f\right\rangle\right| \leqslant C a^{\frac{3}{4}}\left(a^{\alpha}+\left|t_{1}-u_{1}\right|^{\alpha}\right) .
$$

2. If $f \in C^{(1+\varepsilon) \beta}\left(\Gamma_{\left(u_{1}, 0\right)}(L) ;(0,1)\right)$ then there is a constant $C<\infty$ and a fixed coarse scale $a_{0} \in(0,1)$ such that for all $a \in\left(0, a_{0}\right), \boldsymbol{t} \in \Gamma_{\left(u_{1}, 0\right)}(L / 2)$ and $|s| \geqslant \sqrt{a}$,

$$
\left|\left\langle\psi_{a s t}, f\right\rangle\right| \leqslant C a^{\frac{\beta}{2}+\frac{3}{4}}
$$

3. If $f \in C^{(1+\varepsilon) \beta}\left(\Gamma_{\left(u_{1}, 0\right)}(L) ;(0,1)\right)$ and $0<\gamma<\frac{\varepsilon}{1+\varepsilon}<\frac{1}{2}$ then there is a constant $C<\infty$ and a fixed coarse scale $a_{0} \in(0,1)$ such that for all $a \in\left(0, a_{0}\right), \boldsymbol{t} \in \Gamma_{\left(u_{1}, 0\right)}(L / 2)$ and $|s| \geqslant a^{\gamma}$,

$$
\left|\left\langle\psi_{\text {ast }}, f\right\rangle\right| \leqslant C a^{\beta+\frac{3}{4}} .
$$

Proof. To prove suppositions 2-3 above., we assume without loss of generality that $u_{1}=0$. Let $c \in\left(0, \frac{1}{2}\right)$ to be chosen later depending on which statement we want to prove and $R_{a}=\left[-a^{-c}, a^{-c}\right]^{2}$, then $\mathrm{D}_{a} R_{a}=\left[-a^{1-c}, a^{1-c}\right] \times$ $\left[-a^{\frac{1}{2}-c}, a^{\frac{1}{2}-c}\right]$, and hence $\mathrm{B}_{s} \mathrm{D}_{a} R_{a}$, shrinks to $\{\boldsymbol{0}\}$ while $R_{a} \rightarrow \mathbb{R}^{2}$ as $a \rightarrow 0^{+}$. For each $\boldsymbol{x}=\left(x_{1}, x_{2}\right) \in \mathbb{R}^{2}$, let $\boldsymbol{v}_{\boldsymbol{x}}$ denote the vector $\left(x_{1}, \frac{x_{1}}{-s}\right)$ which makes $\boldsymbol{x}-\boldsymbol{v}_{\boldsymbol{x}}=\left(0, x_{2}+\frac{x_{1}}{s}\right)$. Observe that $x_{2}+\frac{x_{1}}{s}=\frac{w_{1}}{s}$ if and only if $\boldsymbol{x}$ is on the line $\boldsymbol{x}=\mathrm{B}_{s}\left(w_{1}, x_{2}\right)^{T}$. That is, for a fixed $w_{1} \in \mathbb{R}$, the sheared line $\mathrm{B}_{s} \ell_{w_{1}}$ of the vertical line $\ell_{w_{1}}=\left\{\left(w_{1}, x_{2}\right)^{T}: x_{2} \in \mathbb{R}\right\}$ is equal to the set of points $\boldsymbol{x}$ satisfying $x_{2}+\frac{x_{1}}{s}=\frac{w_{1}}{s}$. Consequently, for each $\boldsymbol{x} \in \mathrm{B}_{s} \mathrm{D}_{a} R_{a}=$ $\mathrm{B}_{s}\left(\left[-a^{1-c}, a^{1-c}\right] \times\left[-a^{\frac{1}{2}-c}, a^{\frac{1}{2}-c}\right]\right), \boldsymbol{x} \in \mathrm{B}_{s} \ell_{w_{1}}$ for some $\left|w_{1}\right| \leqslant a^{1-c}$ and so $\left\|\boldsymbol{x}-\boldsymbol{v}_{\boldsymbol{x}}\right\|=\left|x_{2}+\frac{x_{1}}{s}\right|=\left|\frac{w_{1}}{s}\right| \leqslant \frac{a^{1-c}}{|s|}$.

Let $a_{0}<1$ be so small that $\mathrm{B}_{s} \mathrm{D}_{a} R_{a} \subseteq \Gamma_{\mathbf{0}}(L / 2)$ whenever $|s|<2$. Let $\boldsymbol{t} \in \Gamma_{\mathbf{0}}(L / 2)$ and $0<a<a_{0}$. Then $\tau_{-\boldsymbol{t}} f \in C^{(1+\varepsilon) \beta}\left(\Gamma_{\mathbf{0}}(L / 2) ;(0,1)\right)$. Hence, for each $\boldsymbol{x} \in \mathrm{B}_{s} \mathrm{D}_{a} R_{a} \subseteq \Gamma_{\mathbf{0}}(L / 2)$, since $\boldsymbol{v}_{\boldsymbol{x}} \in \Gamma_{\mathbf{0}}(L / 2)$, there exists a polynomial $P_{\boldsymbol{v}_{\boldsymbol{x}}}$ of a degree less than $(1+\varepsilon) \beta$ such that

$$
\left|f(\boldsymbol{x}+\boldsymbol{t})-P_{\boldsymbol{v}_{\boldsymbol{x}}}\left(\boldsymbol{x}-\boldsymbol{v}_{\boldsymbol{x}}\right)\right| \leqslant C\left\|\boldsymbol{x}-\boldsymbol{v}_{\boldsymbol{x}}\right\|^{(1+\varepsilon) \beta} \leqslant C \frac{a^{(1-c)(1+\varepsilon) \beta}}{|s|^{(1+\varepsilon) \beta}} .
$$


If $|s| \geqslant a^{1 / 2}$ then $\left|f(\boldsymbol{x}+\boldsymbol{t})-P_{\boldsymbol{v}_{\boldsymbol{x}}}\left(\boldsymbol{x}-\boldsymbol{v}_{\boldsymbol{x}}\right)\right| \leqslant C \frac{a^{(1-c)(1+\varepsilon) \beta}}{a^{(1+\varepsilon) \beta / 2}}=C a^{(1 / 2-c)(1+\varepsilon) \beta}$. Choosing $c=\frac{\varepsilon}{2(1+\varepsilon)}$, we have $\left|f(\boldsymbol{x}+\boldsymbol{t})-P_{\boldsymbol{v}_{\boldsymbol{x}}}\left(\boldsymbol{x}-\boldsymbol{v}_{\boldsymbol{x}}\right)\right| \leqslant C a^{\beta / 2}$. While if we instead assume $|s| \geqslant a^{\gamma}$ then $\left|f(\boldsymbol{x}+\boldsymbol{t})-P_{\boldsymbol{v}_{\boldsymbol{x}}}\left(\boldsymbol{x}-\boldsymbol{v}_{\boldsymbol{x}}\right)\right| \leqslant C a^{(1-c-\gamma)(1+\varepsilon) \beta}$. Choosing $c=\frac{\varepsilon}{1+\varepsilon}-\gamma$, we have $\left|f(\boldsymbol{x}+\boldsymbol{t})-P_{\boldsymbol{v}_{\boldsymbol{x}}}\left(\boldsymbol{x}-\boldsymbol{v}_{\boldsymbol{x}}\right)\right| \leqslant C a^{\beta}$.

This inequality on $\mathrm{B}_{s} \mathrm{D}_{a} R_{a}$ for $|s| \geqslant a^{\gamma}$ yields the estimate:

$$
\begin{aligned}
\left|\int_{\mathrm{B}_{s} \mathrm{D}_{a} R_{a}}\left(f(\boldsymbol{x}+\boldsymbol{t})-P_{\boldsymbol{v}_{\boldsymbol{x}}}\left(\boldsymbol{x}-\boldsymbol{v}_{\boldsymbol{x}}\right)\right) \psi_{a s} \mathbf{0}(\boldsymbol{x}) d \boldsymbol{x}\right| & \leqslant C \int_{\mathrm{B}_{s} \mathrm{D}_{a} R_{a}} \frac{a^{\beta} a^{-\frac{3}{4}}}{1+\left\|\mathrm{D}_{\frac{1}{a}} \mathrm{~B}_{-s} \boldsymbol{x}\right\|^{2 N}} d \boldsymbol{x} \\
& \leqslant C a^{\beta} \int_{R_{a}} \frac{a^{\frac{3}{4}}}{1+\|\boldsymbol{y}\|^{2 N}} d \boldsymbol{y} \\
& \leqslant C a^{\beta+\frac{3}{4}}
\end{aligned}
$$

where Lemma 2.2 is used in the inequality (6). If we only assume $|s| \geqslant a^{1 / 2}$, similar arguments yield $\left|\int_{\mathrm{B}_{s} \mathrm{D}_{a} R_{a}}\left(f(\boldsymbol{x}+\boldsymbol{t})-P_{\boldsymbol{v}_{\boldsymbol{x}}}\left(\boldsymbol{x}-\boldsymbol{v}_{\boldsymbol{x}}\right)\right) \psi_{a s \mathbf{0}}(\boldsymbol{x}) d \boldsymbol{x}\right| \leqslant C a^{\frac{\beta}{2}+\frac{3}{4}}$.

By the decay estimate of $\psi_{\text {as } \mathbf{0}}$ (Lemma 2.2) and a change of variable $(\boldsymbol{x}=$ $\left.\mathrm{B}_{s} \mathrm{D}_{a} \boldsymbol{y}\right)$, if $M$ is an upper bound of $|f(\cdot+\boldsymbol{t})|$ then the integral on $\mathbb{R}^{2} \backslash \mathrm{B}_{s} \mathrm{D}_{a} R_{a}$ can be bounded by

$$
\begin{aligned}
& \mid \int_{\mathbb{R}^{2} \backslash \mathrm{B}_{s} \mathrm{D}_{a} R_{a}}\left(f(\boldsymbol{x}+\boldsymbol{t})-P_{\boldsymbol{v}_{\boldsymbol{x}}}\left(\boldsymbol{x}-\boldsymbol{v}_{\boldsymbol{x}}\right) \psi_{a s \mathbf{0}}(\boldsymbol{x}) d \boldsymbol{x} \mid\right. \\
& \quad \leqslant C a^{-\frac{3}{4}} \int_{\mathbb{R}^{2} \backslash \mathrm{B}_{s} \mathrm{D}_{a} R_{a}} \frac{\left|f(\boldsymbol{x}+\boldsymbol{t})-P_{\boldsymbol{v}_{\boldsymbol{x}}}\left(\boldsymbol{x}-\boldsymbol{v}_{\boldsymbol{x}}\right)\right|}{1+\left\|\mathrm{D}_{\frac{1}{a}} \mathrm{~B}_{-s} \boldsymbol{x}\right\|^{2 N}} d \boldsymbol{x} \\
& \quad \leqslant C a^{\frac{3}{4}} \int_{\mathbb{R}^{2} \backslash R_{a}} \frac{M+P_{\boldsymbol{y}^{\prime}}\left(C^{\prime}\|\boldsymbol{y}\|\right)}{1+\|\boldsymbol{y}\|^{2 N}} d \boldsymbol{y} \quad\left(\text { for some } C^{\prime}>0\right) \\
& \quad=C a^{\frac{3}{4}+c\left(2 N-1-\operatorname{degree} P_{\boldsymbol{y}^{\prime}}\right)}=C a^{K}
\end{aligned}
$$

where $\boldsymbol{y}^{\prime}=\boldsymbol{v}_{\mathrm{B}_{s} \mathrm{D}_{a} \boldsymbol{y}}$ and $K$ can be chosen arbitrarily large as $c$ is fixed and $N$ is arbitrary.

The singularity lines $\Gamma_{\boldsymbol{u}}$ investigated in Theorem 3.2 are always vertical. When one wants to consider singularity along a non-vertical line $\Gamma_{\boldsymbol{u}, s_{0}}$, the following lemma from [27, Lemmas $5.1 \& 5.2]$ is essential.

Lemma 3.3. Let $\boldsymbol{u}=\left(u_{1}, u_{2}\right) \in \mathbb{R}^{2}, s_{0} \in[-2,2], L>0$ and $f$ be a bounded function. Denote $\boldsymbol{u}^{\prime}=\left(u_{1}+s_{0} u_{2}, 0\right)$.

1. If $f \in C^{\alpha}\left(\Gamma_{\boldsymbol{u}, s_{0}}, \Gamma_{\boldsymbol{u}, s_{0}}(L) ;(1,0)\right)$ then $f \circ \mathrm{B}_{s_{0}} \in C^{\alpha}\left(\Gamma_{\boldsymbol{u}^{\prime}}, \Gamma_{\boldsymbol{u}^{\prime}}(L) ;(1,0)\right)$.

2. If $f \in C^{\alpha}\left(\Gamma_{\boldsymbol{u}, s_{0}}(L) ; \mathrm{B}_{s_{0}}(0,1)\right)$ then $f \circ \mathrm{B}_{s_{0}} \in C^{\alpha}\left(\Gamma_{\boldsymbol{u}^{\prime}}(L) ;(0,1)\right)$. 
Applying the lemma, Theorem 3.2 can be generalized to the case with a sheared singularity line, which can be shown by applying the theorem to $f \circ \mathrm{B}_{s_{0}}$ and using the fact that $\left\langle\psi_{a s t}, f\right\rangle=\left\langle\psi_{a\left(s-s_{0}\right) \mathrm{B}_{-s_{0}} t}, f \circ \mathrm{B}_{s_{0}}\right\rangle$.

Theorem 3.4. Let $0<\alpha \leqslant 1, \beta>0$ and $0<\varepsilon<1$ be such that $(1+\varepsilon) \beta \notin \mathbb{N}$. Suppose $f: \mathbb{R}^{2} \rightarrow \mathbb{R}$ is bounded and let $s_{0} \in[-2,2], \boldsymbol{u}=\left(u_{1}, u_{2}\right) \in \mathbb{R}^{2}$ and $L>0$ be given.

1. If $f \in C^{\alpha}\left(\Gamma_{\boldsymbol{u}, s_{0}}, \Gamma_{\boldsymbol{u}, s_{0}}(L) ;(1,0)\right)$ then there exists a constant $C<\infty$ such that for all $a \in(0,1), \boldsymbol{t} \in \mathbb{R}^{2}$ and $\left|s-s_{0}\right| \leqslant \sqrt{a}$,

$$
\left|\left\langle\psi_{\text {ast }}, f\right\rangle\right| \leqslant C a^{\frac{3}{4}}\left(a^{\alpha}+\left|t_{1}+s_{0} t_{2}-u_{1}-s_{0} u_{2}\right|^{\alpha}\right) .
$$

2. If $f \in C^{(1+\varepsilon) \beta}\left(\Gamma_{\boldsymbol{u}, s_{0}}(L) ; \mathrm{B}_{s_{0}}(0,1)\right)$ and $0<\gamma<\frac{\varepsilon}{1+\varepsilon}<\frac{1}{2}$ then there are a constant $C<\infty$ and a fixed coarse scale $a_{0} \in(0,1)$ such that for all $a \in\left(0, a_{0}\right)$ and $\boldsymbol{t} \in \Gamma_{\boldsymbol{u}, s_{0}}(L / 2)$,

$$
\left|\left\langle\psi_{\text {ast }}, f\right\rangle\right| \leqslant \begin{cases}C a^{\frac{\beta}{2}+\frac{3}{4}} & \text { if }\left|s-s_{0}\right| \geqslant \sqrt{a}, \\ C a^{\beta+\frac{3}{4}} & \text { if }\left|s-s_{0}\right| \geqslant a^{\gamma} .\end{cases}
$$

As a result of the theorem, we put together a set of directional regularity conditions to model a linear singularity situation, a similar case of which is considered in [25].

Corollary 3.5. Let $0<\alpha \leqslant 1, \boldsymbol{u} \in \mathbb{R}^{d}, s_{0} \in[-2,2]$ and $N, \varepsilon, \gamma>0$ such that $(1+\varepsilon) N \notin \mathbb{N}$ and $\gamma<\frac{\varepsilon}{1+\varepsilon}$. Suppose $f$ is a bounded function in $C^{\alpha}\left(\Gamma_{\boldsymbol{u}, s_{0}}, \Gamma_{\boldsymbol{u}, s_{0}}(L) ;(1,0)\right)$, $C^{N}\left(\Gamma_{\boldsymbol{u}, s_{0}}(L) \backslash \Gamma_{\boldsymbol{u}, s_{0}} ;(1,0)\right)$ and $C^{(1+\varepsilon) N}\left(\Gamma_{\boldsymbol{u}, s_{0}}(L) ; \mathrm{B}_{s_{0}}(0,1)\right)$ for some $L>0$. Then there is a constant $C>0$ and $a_{0}<1$ such that for all $0<a<a_{0}$, $\boldsymbol{t} \in \Gamma_{\boldsymbol{u}, s_{0}}(L / 2)$ and $s \in[-2,2]$

$$
\left|\left\langle\psi_{\text {ast }}, f\right\rangle\right| \leqslant \begin{cases}C a^{\alpha+\frac{3}{4}} & \text { if } s=s_{0} \text { and } \boldsymbol{t} \in \Gamma_{\boldsymbol{u}, s_{0}} \\ C a^{N+\frac{3}{4}} & \text { if } s=s_{0} \text { and } \boldsymbol{t} \notin \Gamma_{\boldsymbol{u}, s_{0}} \\ C a^{N+\frac{3}{4}} & \text { if }\left|s-s_{0}\right| \geqslant a^{\gamma}\end{cases}
$$

Consequently, for $s=s_{0}$ and $\boldsymbol{t} \in \Gamma_{\boldsymbol{u}, s_{0}},\left\langle\psi_{\text {ast }}, f\right\rangle=O\left(a^{\alpha+\frac{3}{4}}\right)$ as a $\rightarrow 0^{+}$while for all other cases $\left\langle\psi_{\text {ast }}, f\right\rangle=O\left(a^{N+\frac{3}{4}}\right)$ as $a \rightarrow 0^{+}$.

Proof. Let $\boldsymbol{t} \in \Gamma_{\boldsymbol{u}, s_{0}}(L / 2)$. If $s=s_{0}$ and $\boldsymbol{t}$ is on the line $\Gamma_{\boldsymbol{u}, s_{0}}$ then $\left|s-s_{0}\right| \leqslant \sqrt{a}$ and $s_{0}\left(t_{2}-u_{2}\right)+\left(t_{1}-u_{1}\right)=0$. So Theorem 3.4 (1.) gives $\left|\left\langle\psi_{\text {ast }}, f\right\rangle\right| \leqslant C a^{\alpha+\frac{3}{4}}$ for all $0<a<1$.

Let $s=s_{0}$ and $\boldsymbol{t} \notin \Gamma_{\boldsymbol{u}, s_{0}}$. In the light of Theorem 3.4 (1.) on the line $\Gamma_{\boldsymbol{t}, s_{0}}$, the assumption that $f \in C^{N}\left(\Gamma_{\boldsymbol{u}, s_{0}}(L) \backslash \Gamma_{\boldsymbol{u}, s_{0}} ;(1,0)\right)$ implies that $\left|\left\langle\psi_{\text {ast }}, f\right\rangle\right| \leqslant C a^{N+\frac{3}{4}}$ for all $0<a<1$. 
If $\left|s-s_{0}\right| \geqslant a^{\gamma}$ then, by Theorem $3.4(2),.\left|\left\langle\psi_{\text {ast }}, f\right\rangle\right| \leqslant C a^{N+\frac{3}{4}}$ for all $0<a<a_{0}$.

It is now left to show that $\left\langle\psi_{\text {ast }}, f\right\rangle=O\left(a^{N+\frac{3}{4}}\right)$ as $a \rightarrow 0^{+}$when $s \neq s_{0}$. In such a case, it is clear that if $0<a \leqslant\left|s-s_{0}\right|^{1 / \gamma}$ then the shearlet transform is bounded by $C a^{N+\frac{3}{4}}$.

In [9], Grohs characterized the wavefront set, defined via decays of Fourier transform, of a tempered distribution in terms of the decay rates of its continuous shearlet transform. Though the possible singularity in their results is far more general than ours, the singularity in our different setting is based on lower Hölder exponents.

\section{ACKNOWLEDGEMENTS}

The author thanks the Centre of Excellence in Mathematics, the Commission on Higher Education, Thailand, for partial funding. We would also like to thank the referees for bringing [9] to our attention and for their many valuable suggestions which helped to improve the presentation of the paper. Last but not least, our thanks go to Mr. Jukrapong Tiammee who pointed out an error just in time for us to correct the manuscript before publication.

\section{References}

[1] A.P. Calderón and A. Zygmund, Local Properties of Solutions of Elliptic Partial Differential Equations, Stud. Math., 20, 171-225, 1961.

[2] E.J. Candès and D.L. Donoho, Continuous Curvelet Transform. I: Resolution of the Wavefront Set, Appl. Comput. Harmon. Anal., 19(2), 162-197, 2005.

[3] E.J. Candès and D.L. Donoho, Continuous Curvelet Transform. II: Discretization and Frames, Appl. Comput. Harmon. Anal., 19(2), 198-222, 2005.

[4] J-M. Combes, A. Grossmann, and P. Tchamitchian, Wavelets. Time-Frequency Methods and Phase Space, Springer-Verlag, 1989.

[5] I. Daubechies, Ten Lectures on Wavelets, SIAM, 1992.

[6] M.N. Do and M. Vetterli, Contourlets: A directional multiresolution image representation, Proc. IEEE Int. Conf. Image Processing, 2002.

[7] D.L. Donoho, Wedgelets: Nearly minimax estimation of edges, Ann. Stat., 27(3), 859-897, 1999.

[8] G.R. Easley, K. Guo, and D. Labate, Analysis of singularities and edge detection using the shearlet transform, Proceedings of SAMPTA'09, Marseille 2009. 
[9] Philipp Grohs, Continuous Shearlet Frames and Resolution of the Wavefront Set, Monatshefte fr Mathematik, to appear.

[10] K. Guo, G. Kutyniok, and D. Labate, Sparse multidimensional representations using anisotropic dilation and shear operators, in Wavelets and Splines: Athens 2005, eds. G. Chen and M.J. Lai, 189-201, Nashboro Press, 2006.

[11] K. Guo and D. Labate, Optimally sparse multidimensional representation using shearlets, SIAM J. Math. Anal., 39(1), 298-318, 2007.

[12] K. Guo and D. Labate, Representation of Fourier integral operators using shearlets, J. Fourier Anal. Appl., 14(3), 327-371, 2008.

[13] K. Guo and D. Labate, Sparse shearlet representation of Fourier integral operators, Electron. Res. Announc. Math. Sci., 14, 7-19, 2008.

[14] K. Guo and D. Labate, Characterization and analysis of edges using the continuous shearlet transform, SIAM Journal on Imaging Sciences, 2(3), 959986, 2009.

[15] K. Guo, D. Labate, and W.-Q. Lim, Edge analysis and identification using the continuous shearlet transform, Appl. Comput. Harmon. Anal., 27(1), 24-46, 2009 .

[16] K. Guo, D. Labate, W.-Q. Lim, G. Weiss, and E. Wilson, Wavelets with composite dilations, Electron. Res. Announc. Am. Math. Soc., 10, 78-87, 2004.

[17] K. Guo, D. Labate, W.-Q. Lim, G. Weiss, and E. Wilson, Wavelets with composite dilations and their MRA properties, Appl. Comput. Harmon. Anal., 20(2), 202-236, 2006.

[18] M. Holschneider and Ph. Tchamitchian, Pointwise analysis of Riemann's "nondifferentiable" function, Invent. Math., 105(1), 157-175, 1991.

[19] S. Jaffard, Pointwise smoothness, two-microlocalization and wavelet coefficients, Publicacions Matemàtiques, 35, 155-168, 1991.

[20] S. Jaffard, Some mathematical results about the multifractal formalism for functions, in Wavelets: Theory, Algorithms, and Applications, eds. C. Chui K. et al., 321-361, Academic Press, 1994.

[21] S. Jaffard, Local behavior of Riemann's function, in Harmonic Analysis and Operator Theory. A Conference in Honor of Mischa Cotlar. Contemp. Math. 189, eds. S. A. M. Marcantognini et al., 287-307, American Mathematical Society, 1995 .

[22] S. Jaffard, The spectrum of singularities of Riemann's function, Rev. Mat. Iberoam. 12(2), 441-460, 1996. 
[23] S. Jaffard, Multifractal functions: Recent advances and open problems, Bulletin de la Société Royale des Sciences de Liége, 73, 129-153, 2004.

[24] S. Jaffard, Pointwise regularity criteria, C. R., Math., Acad. Sci. Paris, 339, 757-762, 2004.

[25] G. Kutyniok and D. Labate, Resolution of the wavefront set using continuous shearlets, Trans. Amer. Math. Soc., 361(5), 2719-2754, 2009.

[26] D. Labate, W.-Q. Lim, G. Kutyniok, and G. Weiss, Sparse multidimensional representation using shearlets, in Wavelets XI (San Diego, CA, 2005), 254-262, SPIE, 2005.

[27] P. Lakhonchai, J. Sampo, S. Sumetkijakan, Shearlet transforms and directional regularities, International Journal of Wavelets, Multiresolution and Information Processing, 8(5), 743-771, 2010.

[28] S. Mallat, A Wavelet Tour of Signal Processing, Academic Press, 1997.

[29] Y. Meyer, Wavelets and Operators, Hermann, 1990.

[30] K. Nualtong and S. Sumetkijakan, Analysis of Hölder regularity by waveletlike transforms with parabolic scaling, Thai Journal of Mathematics, 3(2), 275$283,2005$.

[31] E.L. Pennec and S. Mallat, Bandelet image approximation and compression, Multiscale Model. Simul. 4(3), 992-1039, 2005.

[32] J. Sampo and S. Sumetkijakan, Estimations of Hölder regularities and direction of singularity by Hart Smith and curvelet transforms, J. Fourier Anal. Appl., 15(1), 58-79, 2009.

[33] H.F. Smith, A Hardy space for Fourier integral operators, J. Geom. Anal., 8(4), 629-653, 1998.

[34] S. Yi, D. Labate, G.R. Easley, and H. Krim, A shearlet approach to edge analysis and detection, IEEE Transactions on Image Processing, 18(5), 929-941, 2009. 